\section{Localized scleroderma and hemiatrophy in association with antibodies to double-stranded DNA}

Sir,

The report of Dr Adebajo and colleagues ${ }^{1}$ includes a further report of a patient with facial hemiatrophy and localized scleroderma, an association which has been previously reported ${ }^{2,3}$ and which invites speculation as to a possible shared pathogenetic mechanism. Such a mechanism may also be applicable to certain patients with 'idiopathic' Parry-Romberg hemifacial atrophy (PRHFA), the aetiology of which has in the past prompted much speculation.

Reporting on a boy with mild hemifacial atrophy, localized leg and trunk scleroderma, and positive antinuclear antibodies, Lewkonia \& Lowry ${ }^{3}$ commented that impairment of facial growth in PRHFA appeared analogous to the effect of linear scleroderma in the limbs. The clinical overlap of localized scleroderma and PRHFA prompted them to conclude that the two syndromes were nosologically related and that PRHFA was no more than an anatomically limited form of localized scleroderma without limb or trunk involvement, rather than a developmental anomaly or dysplasia. If this theory is correct, it will be interesting to learn whether 'idiopathic' PRHFA patients have antibodies to doublestranded DNA. ${ }^{1}$ However, it should be remembered that PRHFA is a clinically heterogeneous condition, and this may reflect aetiological heterogeneity.

Andrew J. Larner The Midland Centre for Neurosurgery \& Neurology, Holly Lane, Smethwick, Warley, West Midlands B67 7JX

\section{References}

1. Adebajo, A.O., Crisp, A.J., Nicholls, A. \& Hazleman, B.L. Localized scleroderma and hemiatrophy in association with antibodies to double-stranded DNA. Postgrad Med J 1992, 68: 216-218.

2. Wartenberg, R. Progressive facial hemiatrophy. Arch Neurol Psychiat 1945, 54: 75-97.

3. Lewkonia, R.M. \& Lowry, R.B. Progressive hemifacial atrophy (Parry-Romberg syndrome). Report with review of genetics and nosology. Am J Med Genet 1983, 14: 385-390. 